\title{
Quantum Hall Ferromagnet in Magnetically-Doped Quantum Wells
}

\author{
T. AndrearczYK ${ }^{a, b}$, J. JAROSZYŃSkI ${ }^{a, b}$, J. WróbeL ${ }^{a}$, \\ G. Karczewski ${ }^{a}$, T. Wojtowicz ${ }^{a}$, E. PaPis ${ }^{c}$, E. Kamińska ${ }^{c}$, \\ A. Piotrowska ${ }^{c}$, D. Popović ${ }^{b}$ And T. Diet ${ }^{a}$ \\ ${ }^{a}$ Institute of Physics, Polish Academy of Sciences \\ al. Lotników 32/46, 02-668 Warszawa, Poland \\ ${ }^{b}$ National High Magnetic Field Laboratory, Florida State University \\ 1800 E. Paul Dirac Dr., Tallahassee, FL 32310, USA \\ ${ }^{c}$ Institute of Electron Technology \\ al. Lotników 32/46, 02-668 Warszawa, Poland
}

\begin{abstract}
The article reviews our recent studies on quantum Hall ferromagnetism in diluted magnetic semiconductors. We carried out magnetoresistance studies on modulation-doped, gated heterostructures of (Cd, Mn) Te/(Cd, Mg) Te:I We put into evidence the formation of Ising quantum Hall ferromagnet with Curie temperature $T_{\mathrm{C}}$ as high as $2 \mathrm{~K}$. Quantum Hall ferromagnetism is manifested by anomalous magnetoresistance maxima. Moreover, magnitude of these spikes depends dramatically on the history of the sample, shows hysteresis when either magnetic field or gate voltage are swept, stretched-exponential time evolution characteristic of glassy systems, and strong Barkhausen noise. Our study suggests that these metastabilities stem from the slow dynamics of ferromagnetic domains.
\end{abstract}

PACS numbers: 73.43.Nq, 72.25.Dc, 73.61.Ga, 75.50.Pp

\section{Introduction}

Two-dimensional electron systems (2DES) under low temperatures $(T)$ and high magnetic field $(B)$ offer a great opportunity for studying the fascinating strong-correlation physics. The carrier density can be controlled externally in 2DES, while tilted $B$ allows one to discriminate between orbital and spin effects. Furthermore, owing to the quenching of the in-plane kinetic energy into quantized 
Landau levels (LL), correlation effects dominate over single particle potentials in a strong magnetic field leading to an unexpectedly rich variety of ground states and quasiparticle forms [1]. In particular, if LL corresponding to the opposite spin orientations of quasi-particles at Fermi level overlap, the spin degree of freedom is not frozen by the field so that a spontaneous spin order may appear at low temperatures [2,3], the resulting state being known as the quantum Hall ferromagnet (QHF). Importantly, the ground state is predicted to have the uniaxial anisotropy if the spin subbands involved originate from different LL [4]. The level arrangement corresponding to such Ising QHF has been realized in various III-V 2DES [5-8].

In recent years we have witnessed a growing interest in diluted magnetic semiconductors (DMS) [9] because of their possible application in spintronics. Previous works demonstrate a strong influence of localized $d$-spins on electron transport of 1D [10,11] and 2D [12] structures of DMS. In DMS the mean-field part of the $s p-d$ exchange interaction between the electrons and $d$-spins leads to the giant $s-d$ spin-splitting of the electronic bands. Since in DMS the spin-splitting of electronic states is not only giant but depends, in a nonlinear fashion, on the magnetic field $B$, many crossings of Landau spin sublevels occur in the quantum Hall effect (QHE) regime, so that in an independent-electron picture, energies come into "coincidence" for particular values of $B$. This worthwhile possibility was explored in the recent study [13], which demonstrated the existence of a quantum Hall ferromagnetism in a II-VI $(\mathrm{Cd}, \mathrm{Mn}) \mathrm{Te} /(\mathrm{Cd}, \mathrm{Mg}) \mathrm{Te}$ modulation doped heterostructure with a quantum well $(\mathrm{QW})$ containing magnetic ions. On either side of these coincidences, the magnetization of the electron system thus takes on distinct values, corresponding to QH ferromagnets (or "Ising" states) of various strengths. The transitions between these ferromagnets provoke sharp spikes in the magnetoresistance. These new peaks are distinct from the usual Shubnikov-de Haas (SdH) maxima between QHE minima. There is a common agreement now concerning the origin of these spikes [14]. In the region of LL coincidence, owing to differences in the local potential landscape, domains of different Ising ferromagnets coexist. Domain walls constitute one-dimensional conducting channels, which make scattering between edge channels possible. Thus longitudinal resistance $\rho_{x x}$ is no longer zero, as it should be in the QHE regime. Accordingly, the magnitude of these spikes increases with the length of domain walls.

In our $(\mathrm{Cd}, \mathrm{Mn}) \mathrm{Te} /(\mathrm{Cd}, \mathrm{Mg}) \mathrm{Te}$ heterostructures the critical behavior of the spike resistance was found, verifying the recent theoretical prediction [14] and making it possible to determine the phase diagram of a $\mathrm{QHF}$ as a function of the carrier density. At the same time, the Curie temperature $T_{\mathrm{C}}$ was found to reach $2 \mathrm{~K}$, a value much higher than that observed and explained theoretically in the case of both (i) high electron mobility AlAs QW in the quantum Hall effect regime $\left(T_{\mathrm{C}} \leq 0.5 \mathrm{~K}[8,14]\right)$ and (ii) $n$-type DMS at $B=0\left(T_{\mathrm{C}} \leq 0.2 \mathrm{~K}\right.$ in $(\mathrm{Zn}, \mathrm{Mn}) \mathrm{O}: \mathrm{Al}$ epilayers [15]). This enhanced stability of the QHF phase is rather surprising in 
view of both previous results $[8,14]$ and the significance of disorder in the present material.

Moreover, the magnitude of the spikes at low temperatures depends strongly on magnetic field and gate voltage $V_{\mathrm{g}}$ sweep direction and rate, leading to a history and time dependent resistance. While there is a common agreement that this metastability constitutes a clear indication that the peaks occur at the magnetic phase transition, a particular mechanism that leads to such a behavior remains a subject of a debate. There are indications that metastability reflects interaction with nuclear spins $[16,17]$ or just a dynamics of ferromagnetic domains [7, 18]. Furthermore, there are theoretical predictions [19] that, in the case when disorder is strong enough, spin-glass rather than ferromagnet forms. In order to shed some new light on this issue, we have undertaken a systematic study of the time-dependent resistance in the regions of ferromagnetic transitions.

\section{Samples}

The modulation-doped $\mathrm{Cd}_{1-x} \mathrm{Mn}_{x}$ Te QW was grown by molecular beam epitaxy [20]. Barriers of $\mathrm{Cd}_{0.8} \mathrm{Mg}_{0.2}$ Te were separated from (001) undoped GaAs substrate by $1 \mathrm{~nm}$ ZnTe and $3 \mu \mathrm{m}$ CdTe buffer layers. The Mn ions were inserted into the QW by the digital alloy technique, i.e. by depositing three evenly spaced monolayers of $\mathrm{Cd}_{1-x} \mathrm{Mn}_{x}$ Te during the growth of $10 \mathrm{~nm}$ wide CdTe QW. According to the spectral position of the photoluminescence line at $77 \mathrm{~K}$, average $x=$ $2 \pm 0.5 \%$. The iodine donors were introduced to the front barrier at $20 \mathrm{~nm}$ away from the QW. For magnetotransport measurements a $0.5 \times 1 \mathrm{~mm}^{2}$ Hall bar was etched, to which leads were soldered with indium. A gold on chromium front gate was deposited onto cap layer. The gate makes it possible to control the electron density $n_{s}$ between 1.4 and $4 \times 10^{11} \mathrm{~cm}^{-2}$, as determined by Hall measurements at low $T$. The low-temperature electron mobility peaks at $\mu \approx 2.5 \times 10^{4} \mathrm{~cm}^{2} /(\mathrm{V} \mathrm{s})$.

\section{Experimental results}

\subsection{Magnetoresistance}

Figure 1a presents the Hall $\rho_{x y}$ and the longitudinal $\rho_{x x}$ resistivities, the latter revealing the presence of a strong resistance "spike" in the magnetic field $B_{c} \approx 5.8 \mathrm{~T}$, which corresponds to the LL filling factor $\nu \equiv n_{s} h /(e B) \approx 2$. According to Fig. $1 \mathrm{~b}$ and $\mathrm{c}$, the $\rho_{x x}$ anomalies peak around $1.3 \mathrm{~K}$. Moreover, at the same $T$ a dramatic shift of the SdH maxima is clearly visible in Fig. 1b. Guided by the previous experimental [8] and theoretical [14] results, we presume that the spikes appear at the crossing points of spin sublevels. In order to evaluate the field values $B_{c}$ at which crossings are expected in our system, we start from the known form of the LL energy in DMS [9] 

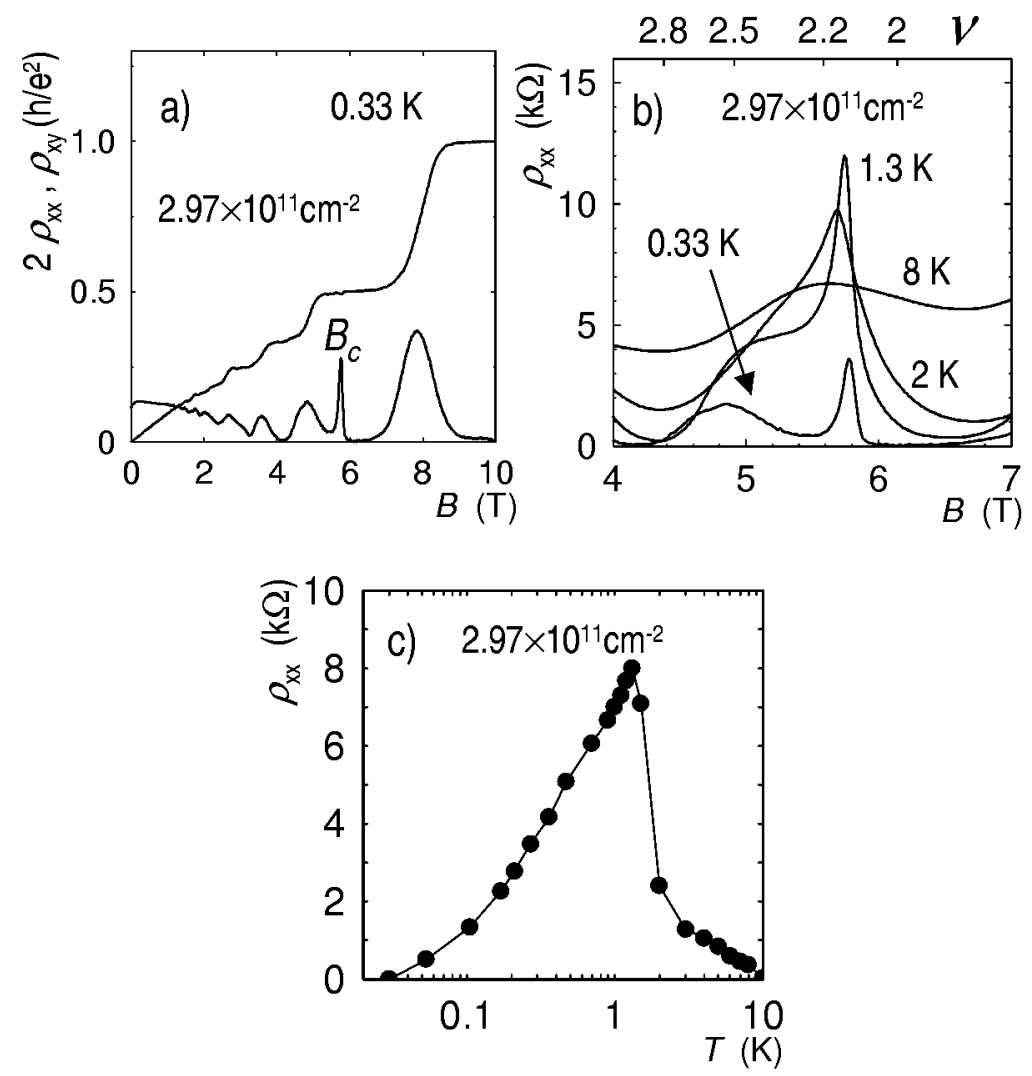

Fig. 1. (a) Resistances $\rho_{x x}$ and $\rho_{x y}$ at $T=0.33 \mathrm{~K}$ for $n_{s}=2.97 \times 10^{11} \mathrm{~cm}^{-2}\left(V_{\mathrm{g}}=0\right)$. Note the presence of a spike in $\rho_{x x}$ at $B_{c} \simeq 5.8 \mathrm{~T}$, shown at selected temperatures in (b). (c) The spike height as a function of $T$ for $n_{s}=2.97 \times 10^{11} \mathrm{~cm}^{-2}$. If the spike and the $\mathrm{SdH}$ peak overlap, the contribution of the latter is subtracted.

$$
\begin{aligned}
E_{n, \uparrow, \downarrow} & =(n+1 / 2) \hbar e B \cos \theta / m^{*} \\
& \pm \frac{1}{2}\left[g^{*} \mu_{\mathrm{B}} B+\alpha N_{0} x_{\mathrm{eff}} S B_{S}\left(\frac{S g \mu_{\mathrm{B}} B}{k_{\mathrm{B}}\left[T+T_{\mathrm{AF}}\right]}\right)\right] .
\end{aligned}
$$

Here $n$ is the LL index, $m^{*}=0.10 m_{0}$ and $g^{*}=-1.67$ [21] are the effective mass and Landé factor of the electrons in CdTe, $\alpha N_{0}=0.22 \mathrm{eV}$ is the $s-d$ exchange energy [9, 22], and $B_{S}$ is the Brillouin function, in which $S=5 / 2$ and $g=2.0$. The functions $x_{\mathrm{eff}}(x)<x$ and $T_{\mathrm{AF}}(x)>0$ [22] describe the reduction of magnetization $M(T, H)=g \mu_{\mathrm{B}} x_{\mathrm{eff}} N_{0} S B_{S}(T, H)$ by antiferromagnetic interactions.

We take into account correlation energy determined experimentally [8] and theoretically [14] for the AlAs QW. As shown in Fig. 2a and b, we obtain a remarkably good description of the spike positions $B_{c}$ with one adjustable parameter, the Mn concentration $x$ set at $1.37 \%$, for which $x_{\mathrm{eff}}=1.12 \%$ and $T_{\mathrm{AF}}=0.47 \mathrm{~K}$. 

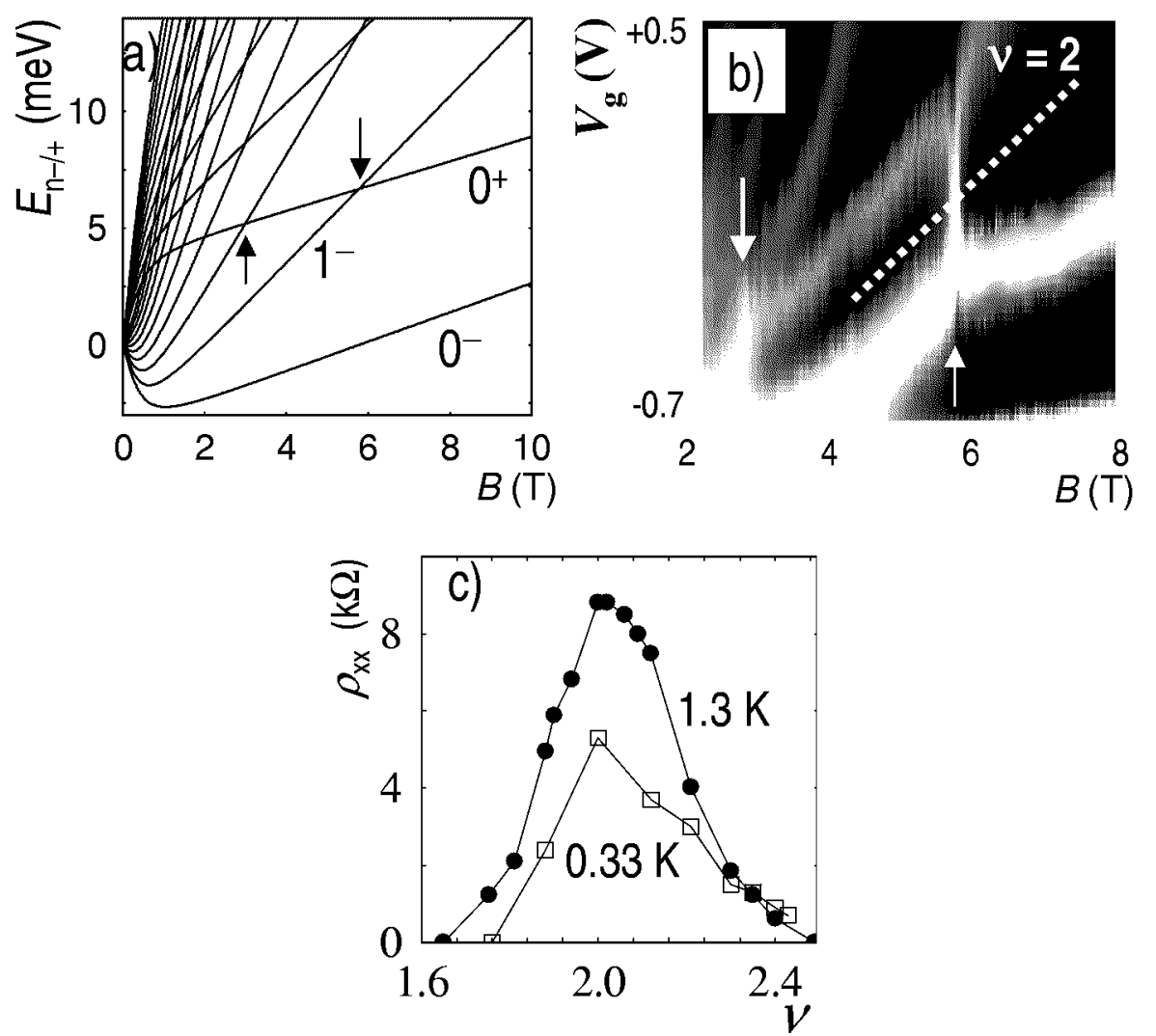

Fig. 2. (a) Diagram of the Landau energy levels in a magnetic quantum well calculated from Eq. (1), showing the spin-up and spin-down levels intersecting in a perpendicular magnetic field. (b) Longitudinal resistivity as a function of the magnetic field and gate voltage. Bright (dark) regions represent high (low) resistivity. (c) The height of the QHF spike at $B_{c} \simeq 5.8 \mathrm{~T}$ as a function of the filling factor. Arrows show intersections of $0^{+}$ with $1^{-}$and $2^{-}$Landau levels in (a) and resulting longitudinal resistance anomalies in (b).

The interpretation of the resistance spikes in terms of the QHF formation implies that they should disappear if $\nu$ deviates from an integer at $B_{c}$. This is clearly demonstrated in Fig. 2c, where the magnitude of the spike is presented as a function of the filling factor $\nu$ at $B_{c}=5.8 \mathrm{~T}$. Indeed, the spike amplitude peaks at $\nu=2 \pm 0.02$, and it decreases for both smaller and higher $\nu$ values.

\subsection{Hysteretic behavior}

Having established that the spikes occur if LLs cross at $\nu$ close to integers, we turn to experimental evidence that they correspond to a phase transition at nonzero temperatures. According to Fig. 1c, the spike magnitude exhibits a rather 
sharp maximum at the temperature that we identify as $T_{\mathrm{C}}$. At the same time, a hysteresis loop of $\rho_{x x}(B)$ develops when $B$ is swept in two directions below $T_{\mathrm{C}}$ at the precise location of the spike, as presented in Fig. 3a. Hence, our results corroborate the notion [4] that if $\nu$ is close to an integer at $B_{c}$, a transition to Ising QHF ground state takes place. In this broken symmetry state, all electrons fill up one LL, leaving the other one empty. This is evidenced in Fig. 1b which reveals the absence of a $\mathrm{SdH}$ maximum at $B_{c}$ below $T_{\mathrm{C}}$. However, depending on a local potential landscape either $0^{+}$or $1^{-}$LL is filled up in a given space region. Domain walls appearing in this way form edge-like channels. Their presence gives rise to an additional scattering that results in the resistance spike at $B_{c}$ [14].
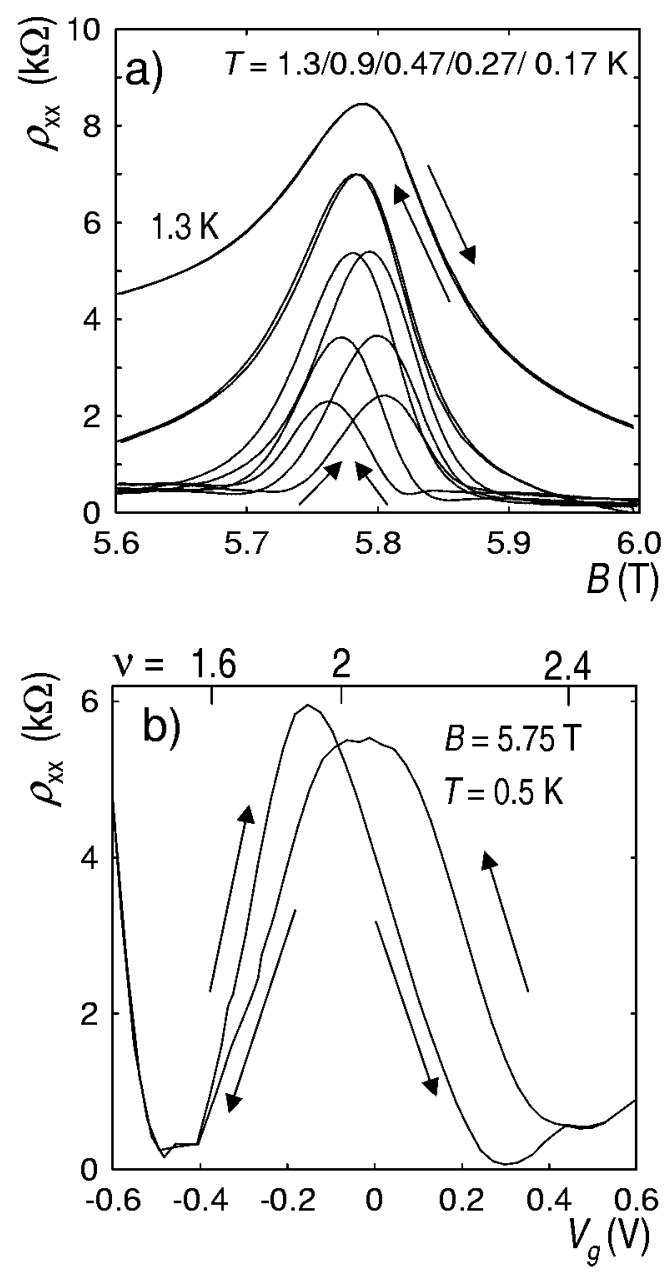

Fig. 3. Hysteresis loops, where $\rho_{x x}$ is depicted in the region of the QHF spike for sweeping the magnetic field (a) and the gate voltage (b) in two directions. 
It is now established that their random configurations below a critical temperature $T_{\mathrm{C}}$ lead to large energy barriers between adjacent domains. This gives rise to metastable states with slow evolution, which are responsible for the observed hysteretic behavior. However, the origin of such a glassy system is a subject of current debate.

Figure $3 \mathrm{~b}$ shows another manifestation of the hysteretic behavior, induced when the gate voltage is tuned in the vicinity of the resistance spike. In this experiment $V_{g}$ was swept between -0.6 and $+0.6 \mathrm{~V}$ at the rate of $0.024 \mathrm{~V} / \mathrm{s}$ at $B=5.75 \mathrm{~T}$ and $T=0.5 \mathrm{~K}$. A number of important conclusions emerge from this measurement. First, a strong hysteresis is observed exclusively in the region of the QHF spike, i.e. for $1.6<\nu<2.4$, while it disappears for both smaller and higher $\nu$ values, that is in the regions of the usual $\mathrm{SdH}$ maxima. Second, hysteresis changes its sign exactly at $\nu=2$ and is stronger for $\nu>2$ than for $\nu<2$.

\subsection{Resistance relaxation}

The hysteretic behavior, observed when either $B$ or $V_{g}$ are tuned, constitutes a strong evidence of the magnetic origin of the resistance spikes. However, in order to extract the time scales involved, it is more convenient to change $B$ or $V_{g}$ abruptly and then study resistance relaxation towards equilibrium. While an instant change of $B$ is practically impossible at low temperatures, this is easy to accomplish with $V_{g}$.

Figure $4 \mathrm{a}$ presents such a relaxation experiment. First, the gate voltage was kept at $V_{g}^{\mathrm{i}}=+0.5 \mathrm{~V}$, which corresponds to $\nu \approx 2.5$, for some waiting time
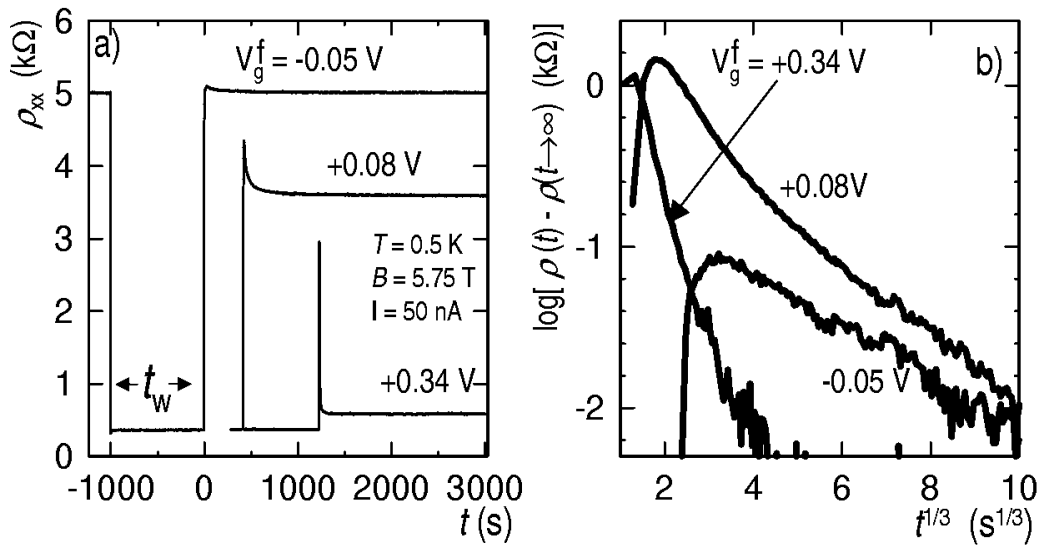

Fig. 4. (a) Traces of $\rho_{x x}(t)$ measured after a sudden change of $V_{g}$ from $V_{g}^{\mathrm{i}}=+0.5 \mathrm{~V}$ $(\nu \approx 2.5)$ to $V_{g}^{\mathrm{f}}=-0.05,+0.08,+0.34(\nu=2.06,2.2,2.4)$. (b) Traces of $\rho_{x x}(t)$ for $V_{g}^{\mathrm{f}}=-0.05,+0.08,+0.34$ corresponding to $\nu>2$ plotted on the scale in which stretched exponential dependence $\exp \left(-(t / \tau)^{1 / 3}\right)$ would be linear. Measurements were performed at $B=5.75 \mathrm{~T}$ after a magnetic field sweep from $B=0(0.24 \mathrm{~K})$ and from $B=9 \mathrm{~T}(0.7$ and $1.4 \mathrm{~K})$. 
$t_{\mathrm{w}} \approx 1000 \mathrm{~s}$. Then the gate voltage was changed instantly to different $V_{g}^{\mathrm{f}}$ around QHF spike, and the time evolution of resistance was recorded for a long time. It is seen that $\rho_{x x}$ first increases fast during the initial $\sim 100 \mathrm{~s}$ and then decreases slowly. These results are consistent with the hysteretic behavior shown in Fig. 3b. Moreover, while this initial increase is exponential, the slow process, as it was shown in Fig. 4b, is described by a stretched exponential function

$$
\rho_{x x}(t)=\rho_{x x}(t \rightarrow \infty) \pm A \exp \left[-(t / \tau)^{s}\right]
$$

with $s \approx 0.33 \pm 0.05$ and increasing time $\tau$ as $\nu \rightarrow 2$. Such a non-Arrhenius behavior is characteristic of electron, spin, and structural glasses with rich energy landscape and thus a wide range of relaxation times.

It should be noted that slow relaxation is observed exclusively at the QHF resistance spikes, while in other regions, such as the usual SdH maxima, it is absent. Thus, it cannot be explained as a similar experiment with a resistance relaxation after a sharp $V_{g}$ change performed in nonmagnetic $\mathrm{In}_{2} \mathrm{O}_{3}$ [23] in which slow electron glass dynamics stems from electron glass formation, without any magnetic effects.

\subsection{Resistance noise}

Further evidence for glassy behavior is shown in Fig. 5, which presents $\rho_{x x}(t)$ traces taken at the QHF spike at various temperatures over a long time period. At the lowest temperature, the average $\rho_{x x}$ decreases slowly with time. However, after some time, distinguishable jumps appear in the resistance trace. Their magnitude increases with time, while their frequency decreases. This Barkhausen-like noise clearly reflects the dynamics of ferromagnetic domains. The domains become bigger with time, thus their walls that are responsible for scattering become shorter and,

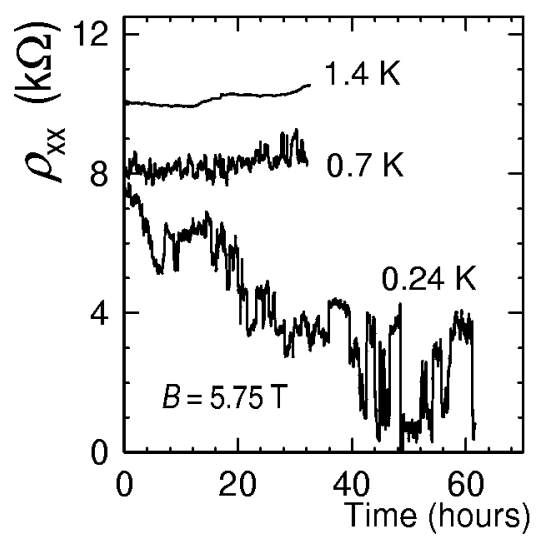

Fig. 5. Traces of $\rho_{x x}(t)$ measured in the region of QHF spike at various temperatures. Measurements were performed at $B=5.75 \mathrm{~T}$ after a magnetic field sweep from $B=0$ $(0.24 \mathrm{~K})$ and from $B=9 \mathrm{~T}(0.7$ and $1.4 \mathrm{~K})$. 
as a result, $\rho_{x x}(t)$ decreases. The amplitude of Barkhausen noise decreases as $T$ increases, and eventually for $T=1.4 \approx T_{\mathrm{C}}=1.3 \mathrm{~K}$, no Barkhausen noise is observed. Therefore, we can connect this noise with the QHF formation not only because it appears exclusively in the region of QHF spikes, but also because it is seen only in the temperature range below $T_{\mathrm{C}}=1.3 \mathrm{~K}$ of the ferromagnetic phase transition.

Slow resistance dynamics could be in principle explained within a framework of many models proposed for this issue, as the relaxation of nuclear spin polarization [16], the formation of nuclear spin glass [17] or the formation of an electronic quantum Hall spin glass [19]. However, the observation of such a pronounced Barkhausen noise strongly supports a model [7, 18] in which the metastable ferromagnetic domains are responsible for slow resistance dynamics observed in the QHF regime.

\section{Acknowledgments}

The work was supported by NSF grant DMR-0071668, NHMFL through NSF Cooperative Agreement DMR-0084173, Center of Excellence CELDIS EU Contract No. ICA1-CT-2000-70018, Polish KBN grants K035-P03-2002 and 7T08A 04721 .

\section{References}

[1] Perspectives in Quantum Hall Effects, Eds. S. Das Sarma, A. Pinczuk, Wiley, New York 1997.

[2] G.F. Giuliani, J.J. Quinn, Phys. Rev. B 31, 6228 (1985).

[3] S.M. Girvin, Physics Today 53(6), 39 (2000) and references therein.

[4] T. Jungwirth, A.H. MacDonald, Phys. Rev. B 63, 035305 (2001).

[5] S. Koch, R.J. Haug, K. v. Klitzing, M. Razeghi, Phys. Rev. B 47, 4048 (1993).

[6] V. Piazza, V. Pellegrini, F. Beltram, W. Wegscheider, T. Jungwirth, A.H. MacDonald, Nature 402, 638 (1999).

[7] J. Eom, H. Cho, W. Kang, K.L. Campman, A.C. Gossard, M. Bichler, W. Wegscheider, Science 289, 2320 (2000).

[8] E.P. De Poortere, E. Tutuc, S.J. Papadakis, M. Shayegan, Science 290, 1546 (2000).

[9] T. Dietl, in: Handbook on Semiconductors, Eds. T.S. Moss, North-Holland, Amsterdam 1994, p. 1251.

[10] J. Jaroszynski, J. Wróbel, M. Sawicki, E. Kaminska, T. Skoskiewicz, G. Karczewski, T. Wojtowicz, A. Piotrowska, J. Kossut, T. Dietl, Phys. Rev. Lett. 75, 3170 (1995).

[11] J. Jaroszynski, J. Wróbel, G. Karczewski, T. Wojtowicz, T. Dietl, Phys. Rev. Lett. 80, 5635 (1998). 
[12] I.P. Smorchkova, N. Samarth, J.M. Kikkawa, D.D. Awschalom, Phys, Rev, Lett. 78, 3571 (1997).

[13] J. Jaroszyński, T. Andrearczyk, G. Karczewski, J. Wróbel, T. Wojtowicz, E. Papis, E. Kamińska, A. Piotrowska, D. Popović, T. Dietl, Phys. Rev. Lett. 89, 266802 (2002).

[14] T. Jungwirth, A.H. MacDonald, Phys. Rev. Lett. 87, 216801 (2001).

[15] T. Andrearczyk, J. Jaroszynski, M. Sawicki, Le Van Khoi, T. Dietl, D. Ferrand, C. Bourgognon, J. Cibert, S. Tatarenko, T. Fukumura, Zhengwu Jin, H. Koinuma, M. Kawasaki, in: Proc. 25th Int. Conf. on Physics of Semiconductors, Osaka 2000, Eds. N. Miura, T. Ando, Springer-Verlag, Berlin 2001, p. 325.

[16] J.H. Smet, R.A. Deutschmann, F. Ertl, W. Wegscheider, G. Abstreiter, K. von Klitzing, Nature 415, 281 (2002).

[17] S. Kraus, O. Stern, J.G.S. Lok, W. Dietsche, K. von Klitzing, M. Bichler, D. Schuh, W. Wegscheider, Phys. Rev. Lett. 89, 266801 (2002).

[18] V. Piazza, V. Pellegrini, F. Beltram, W. Wegscheider, Solid State Commun. 127, 163 (2003).

[19] S. Rapsch, J.T. Chalker, D.K.K. Lee, Phys. Rev. Lett. 88, 036801 (2002).

[20] G. Karczewski, J. Jaroszynski, A. Barcz, M. Kutrowski, T. Wojtowicz, J. Kossut, J. Cryst. Growth 184/185, 814 (1998).

[21] C.Y. Hu, W. Ossau, D.R. Yakovlev, G. Landwehr, Phys. Rev. B 58, R1766 (1998).

[22] J.A. Gaj, W. Grieshaber, C. Bodin-Deshayes, J. Cibert, G. Feuillet, Y. Merle D’Aubigné, A. Wasiela, Phys. Rev. B 50, 5512 (1994).

[23] A. Vaknin, Z. Ovadyahu, M. Pollak, Phys. Rev. Lett. 84, 3402 (2000). 